ORIGINAL ARTICLE

\title{
Expression of secreted Wnt antagonists in gastrointestinal tissues: potential role in stem cell homeostasis
}

\author{
T Byun, M Karimi, J L Marsh, T Milovanovic, F Lin, R F Holcombe
}

J Clin Pathol 2005;58:515-519. doi: 10.1136/icp.2004.018598

See end of article for authors' affiliations

Correspondence to:

Correspondence to:
Dr R F Holcombe, $M D$,

Division of Hematology/

Oncology, University of

California, Irvine Medical

Center, 101 The City

Drive, Bld 23, Rm 244

Orange, CA 92868, USA;

rholcomb@uci.edu

Accepted for publication August 2004
Background: Wnt signalling dysregulation has been implicated in cancer, including colon and gastric cancer. Initiation of Wnt signalling is modulated by soluble Wnt antagonists (sWAs), including soluble frizzled related proteins, dickkopf (Dkk) proteins, and Wnt inhibitory factor-1 (Wif1).

Aims: To evaluate the role of sWAs in upper (gastric) and lower (colon) gastrointestinal tract tumorigenesis.

Methods: Dkk1-3, Wif1, and FrzB expression was evaluated by in situ RNA hybridisation on normal and malignant human gastric and colon tissues. Expression was graded semiquantitatively.

Results: Wif1, Dkk1, and Dkk2 were not expressed in normal gastric tissue. Dkk3 was expressed in some samples, with stronger expression in deep gastric glands. FrzB was expressed in several normal gastric samples, but not in matched tumour specimens. In contrast, Dkkl and FrzB were not expressed in normal colon. Wif1 was expressed in most colon samples, with stronger expression at crypt bases. Dkk3 and Dkk2 expression was also concentrated at crypt bases. There were no differences between sWA expression in malignant colon and matched normal tissue.

Conclusions: sWA expression differed between upper and lower gastrointestinal tract. The loss of FrzB in gastric cancer suggests that it acts as a tumour suppressor. The graded expression of Dkk3 in gastric tissue, and Dkk2, Dkk3, and Wif1 in colon tissue, with increased expression in the deep gastric glands/colonic crypt bases, where gastrointestinal stem cells reside, suggests that sWAs may be crucial Wnt signalling regulators in these tissues, and may contribute to stem cell pool maintenance. sWAs are important components of the gastrointestinal proliferative regulatory network.
$\mathrm{T}$ he Wnt signalling pathway controls patterning and cell fate determination in the development of a wide range of organisms, from drosophila to mammals. ${ }^{1}$ The Wnt genes encode a large family of secreted molecules that play important roles in controlling tissue patterning, cell fate, and cell proliferation within a broad range of embryonic contexts, including the gastrointestinal tract. ${ }^{2}$ To date, 19 members of the Wnt family have been identified in humans, along with 10 members of the frizzled (Fz) family, serpentine transmembrane cell surface receptors through which the Wnt signalling cascade is initiated.

"The Wnt genes encode a large family of secreted molecules that play important roles in controlling tissue patterning, cell fate, and cell proliferation within a broad range of embryonic contexts, including the gastrointestinal tract $^{\prime \prime}$

In the canonical Wnt pathway, binding of Wnt ligands to the $\mathrm{Fz}$ receptors results in activation of disheveled protein and subsequent inhibition of glycogen synthase kinase $3 \beta$. This, in turn, prevents adenomatous polyposis coli and Axin dependant degradation of $\beta$ catenin, leading to its accumulation. The accumulated $\beta$ catenin translocates to the nucleus and binds to members of the lymphoid enhancer factor/T cell factor family of high mobility group box transcription factors, inducing the transcription of growth regulatory genes. There are other, non-canonical pathways including the Wnt-Ca ${ }^{2+}$ pathway and the planar polarity pathway that are less well understood in relation to human carcinogenesis. ${ }^{3-6}$

The interactions of Wnt ligands and $\mathrm{Fz}$ receptors are modulated by the secreted Wnt antagonists (sWAs), which can be divided into two functional classes: the soluble frizzled related protein (sFRP) class and the dickkopf (Dkk) class. Members of the sFRP family include Wnt inhibitory factor- 1 (Wifl), sFRP1, 2, 4, and 5, and frisbee (FrzB, sFRP3). They bind directly to Wnts, thus altering their ability to bind the $\mathrm{Fz}$ receptors. Four members of the Dkk family, Dkkl-4, bind to low density lipoprotein receptor related proteins (LRP5 and 6) contained within the Wnt receptor complex and influence Wnt signalling by preventing normal LRP-Fz-Wnt interactions. $^{7}$

The various secreted Wnt antagonists interact directly and indirectly to affect Wnt signalling and influence a wide variety of biological processes, including developmental cell fate, differentiation, and tumorigenesis. In our study, we have used an in situ, antisense RNA hybridisation technique to examine the expression of selected sWAs and explore any possible associations between sWAs and tumorigenesis in the upper and lower human gastrointestinal tract.

\section{METHODS}

\section{Tissue acquisition}

Archived, paraffin wax embedded pathological specimens were obtained under an IRB approved protocol. Patients were identified through the Chao Family Comprehensive Cancer Center as individuals with surgical resections of colonic or gastric adenocarcinoma. Samples of normal gastric or colon tissue and of cancer tissue were obtained from different cuts of the same surgical specimen for each patient. Fifteen patients with colon cancer and 12 with gastric cancer were enrolled in our study. The histology of the colon cancers was predominately moderately differentiated or moderate to well

Abbreviations: Dkk, dickkopf; FrzB, Frisbee; Fz, frizzled; LRP, lipoprotein receptor related protein; sFRP, soluble frizzled related protein; sWA, soluble Wnt antagonist; Wif1, Wnt inhibitory factor-1 


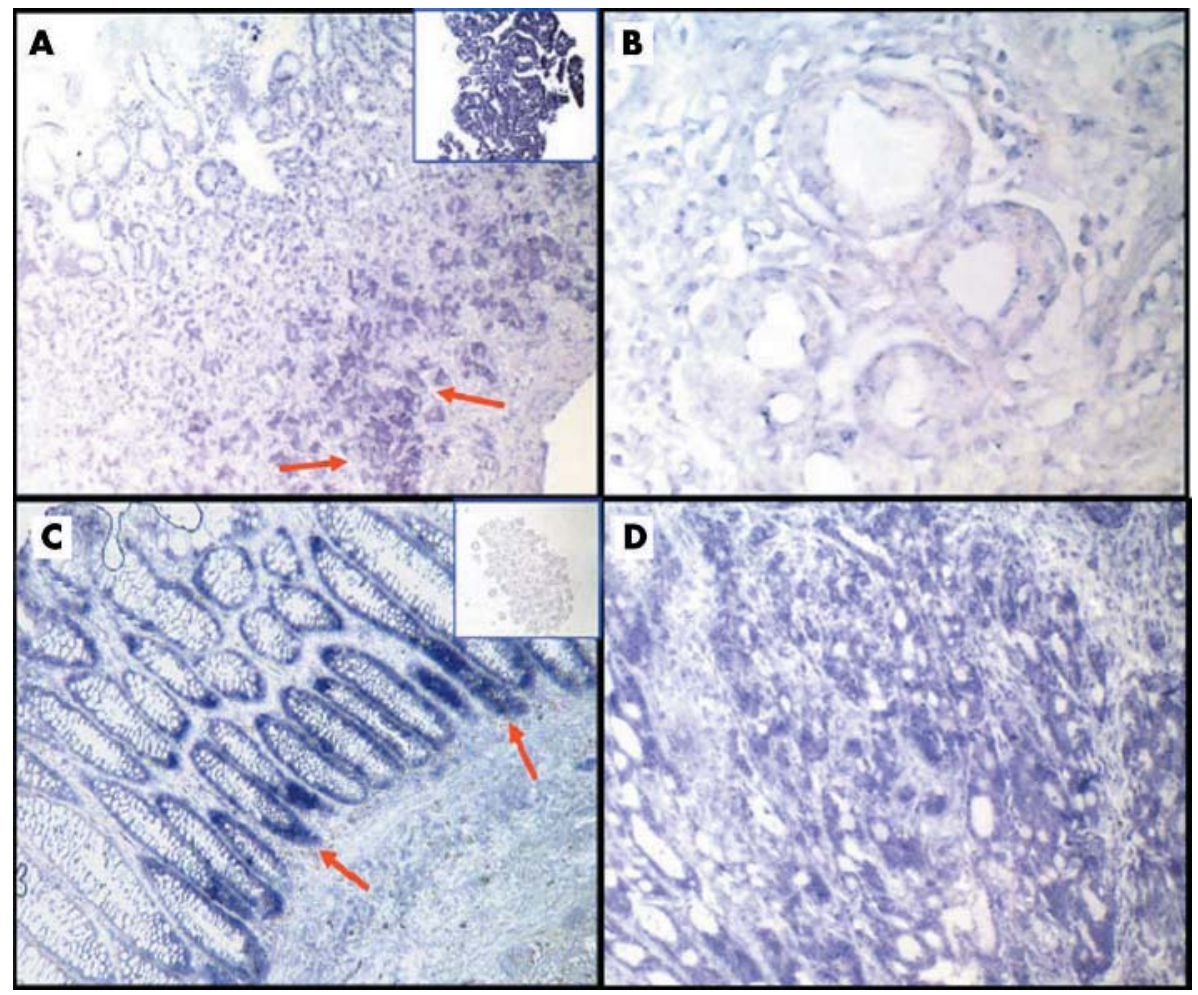

Figure 1 In situ, antisense RNA hybridisation of Dkk3 in gastric and colonic normal and cancer tissue. A dark blue/purple colour indicates positive hybridisation. The insert in panel A shows a positive control with $\mathrm{CaCo} 2$ colon cancer cells, and that in panel $\mathrm{C}$ shows a negative control with HT-29 colon cancer cells. (A) Normal gastric tissue. Note the positive hybridisation with a stronger intensity of expression in deep gastric glands (arrows). Original magnification, $\times 10$. (B) Gastric cancer tissue. Negative hybridisation was found in seven of nine samples tested. FAST red dye was used as a counterstain to reveal tissue architecture. Original magnification, $\times 40$. (C) Normal colon tissue. Note the positive hybridisation with a stronger intensity of expression at the base of the crypts (arrows). Original magnification, $\times 10$. (D) Colon cancer tissue. Positive hybridisation was found in four of 15 samples tested. Original magnification, $\times 10$.

differentiated. Only one sample contained an appreciable amount of poorly differentiated cancer. About half of the patients had lymph node involvement (stage III) at the time of surgical resection and one patient presented with metastatic (stage IV) disease, although the metastatic site was not biopsied. Gastric cancers included well differentiated tumours (three), moderately well differentiated tumours (three; one classified as intestinal type), and poorly differentiated tumours (six; diffuse, intestinal, and signet ring classifications). None of the patients from whom gastric cancer specimens were obtained had evidence of distant metastases at the time of diagnosis.

\section{Cell lines}

Human cell lines were obtained from the American Type Culture Collection (Manassas, Virginia, USA). They comprised HT29, RKO, and Colo205, and were grown in conditioned medium as recommended by the company. The cells were pelleted by centrifugation, embedded in paraffin wax, sectioned, and prepared in a similar manner to the tissue (described below) for in situ RNA hybridisation. These cell lines served as positive and negative controls.

\section{In situ RNA hybridisation}

Tissue slides were dewaxed with sequential xylene/alcohol/ DEPC treated $\mathrm{H}_{2} \mathrm{O}$ washes and hybridised with single stranded antisense RNA probes generated from cDNAs using T7 and T3 RNA polymerase promoters in the respective vectors as described previously. ${ }^{8}$ Briefly, the probes contained sequences encoding $3^{\prime}$ untranslated regions to minimise crossreactivity. Controls included paraffin wax embedded cell lines known to express, or not express, the relevant genes.
Sense orientation RNA probes were used as additional negative controls. Probes were labelled with digoxigenin, hydrolysed, and hybridised to tissue sections in a moisture controlled environment at $37^{\circ} \mathrm{C}$ for 72 hours under conditions optimised for each probe. Positive hybridisation was detected using antidigoxigenin conjugated to alkaline phosphatase to allow visualisation with an alkaline phosphatase substrate. All slides were counterstained with a FAST red reagent to reveal the tissue architecture. Comparisons of normal tissue with malignant tissue on different regions of the same slide ensured identical treatment of tissues for comparison of expression.

Expression was visualised directly by means of an Olympus B50 microscope system with Nomarski optics and digital capture technology. Photographs of tissue and cell line sections were obtained within 72 hours of completion of the hybridisation to minimise slide artefacts, which can appear over time.

Probes used for in situ hybridisation included Wifl, Dkkl, Dkk2, Dkk3, and FrzB obtained from Genome Systems (St Louis, Illinois, USA). The genes and the accession and identification numbers from the Image Consortium are listed as follows: Wifl, 221974, H83294; Dkk1, 471724, AA035443; Dkk2, 1233516, AA715452; Dkk3, 5264858, BI553372; and FrzB, 51061, H18848.

\section{RESULTS}

Expression of sWAs was initially graded as negative (no cells staining), weakly positive (faint staining), or positive (evident staining) by two blinded observers. A third observer was used if any discrepancy existed to generate a majority consensus. For data analysis, the expression pattern was 


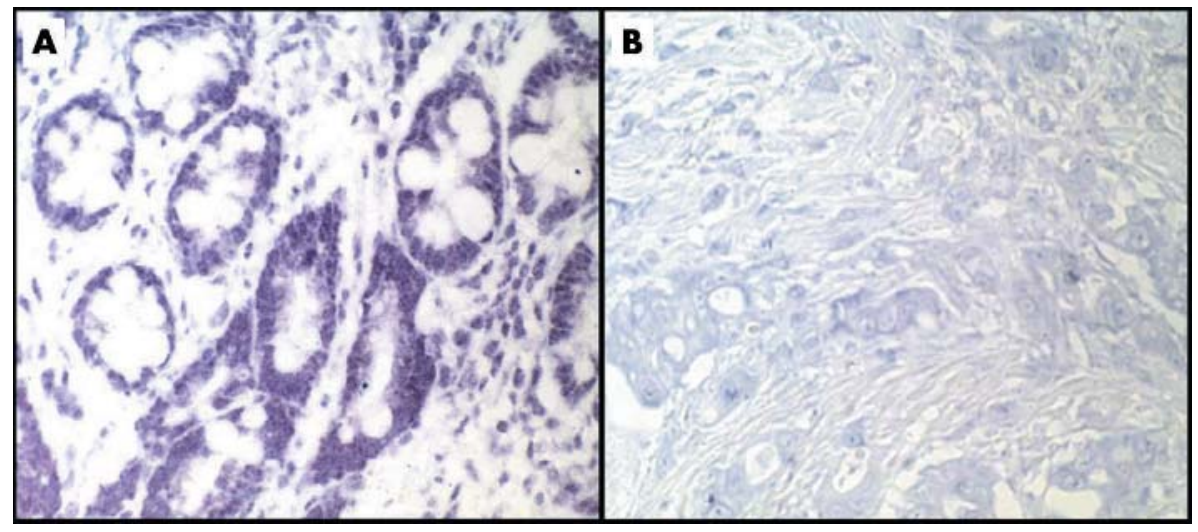

Figure 2 In situ hybridisation using an antisense FrzB RNA probe in gastric tissue samples. The purple colour indicates positive hybridisation. (A) Normal gastric tissue. Positive hybridisation was found in two of five samples tested. Original magnification, $\times 40$. (B) Gastric cancer tissue. Negative hybridisation from the same patient as panel (A). Original magnification, $\times 40$. Normal colon and colon cancer tissue exhibited no staining for FrzB (not shown).

graded as either negative or positive. In addition to this semiquantitative grading, any apparent differences in staining patterns at particular locations within the sample (for example, the base of a colonic crypt) were recorded.

Wifl, Dkk1, and Dkk2 were not expressed in either normal or malignant gastric tissue (table 1). Dkk3 was expressed in four of nine samples of gastric normal tissue and two of nine samples of gastric malignant tissue (fig 1). In normal tissue, there was stronger expression of Dkk3 in the deep gastric glands than in the gastric glands closer to the mucosal surface. We have termed this a "zonal" expression pattern. FrzB was expressed in two of five normal gastric tissue samples, but was absent in all malignant gastric tissue samples tested (fig 2). No correlation was seen with the expression of specific sWAs and the histological grade or descriptive subtype.

Wifl was expressed in most normal and malignant colon tissue samples (table 1). In the normal tissue samples, there was a stronger or zonal expression of Wifl mRNA at the base of colon crypts (fig 3). Dkk3 exhibited similar zonal expression, but was detected in only five of 14 normal and four of 15 malignant samples. Dkk2 was expressed in one of three normal and one of three malignant tissue samples, with zonal expression in the one normal sample showing expression. Dkkl and FrzB were not expressed in either normal or malignant colon tissue. No correlation between the expression of sWAs and the presence of lymph node or metastatic disease at the time of diagnosis (stage III or IV) was seen.

\section{DISCUSSION}

Wnt signalling is known to be involved in gut development in both invertebrate and vertebrate model systems. ${ }^{9-11}$ For

Table 1 Expression of secreted Wnt antagonists in gastric and colon tissue samples

\begin{tabular}{llllll}
\hline Tissue & Wif1 & Dkk1 & Dkk2 & Dkk3 & FrzB \\
\hline Normal gastric & $0 / 5$ & $0 / 5$ & $0 / 5$ & $4 / 9$ & $2 / 5$ \\
Gastric cancer & $0 / 5$ & $0 / 5$ & $0 / 5$ & $(4 / 4)$ & \\
Normal colon & $11 / 11$ & $0 / 5$ & $1 / 3$ & $5 / 14$ & $0 / 5$ \\
Colon cancer & $7 / 5)$ & $0 / 5$ & $(1 / 1)$ & $(5 / 5)$ & \\
& $7 / 3$ & $4 / 15$ & $0 / 9$ \\
\hline
\end{tabular}

Numbers in parenthesis denote tissue samples exhibiting graded or zonal expression of a secreted Wnt antagonist. example, during various stages of gut development in the chick embryo differential expression of Wnt, Fz, and sWA genes suggests that a spatially and temporally regulated matrix of factors influences signalling through the Wnt pathway to promote normal foregut formation. ${ }^{12}{ }^{13}$ In mice, disruption of the Tcf7/2 gene, which encodes the Wnt downstream transcriptional regulatory protein Tcf4, by homologous recombination impairs intestinal development and reduces the intestinal stem cell compartment. ${ }^{14}$

The pattern of sWA expression in the human upper gastrointestinal tract is distinct from that seen in the lower tract. In normal gastric tissues, expression of Dkk3 and FrzB was seen and Wifl and Dkk2 transcripts were not detected. In the normal colon, Wif 1 expression was seen in all samples, and Dkk2 and Dkk3 were expressed in approximately one third of the samples tested. No FrzB expression was seen. Interestingly, no expression of Dkkl was seen in the normal or malignant gastric or the normal or malignant colonic tissues. Transgenic mice that ectopically express Dkkl exhibit a reduction in intestinal epithelial proliferation and loss of intestinal crypts. ${ }^{15}$ Based on our data, at least in adult, human gastrointestinal tissues, Dkkl does not appear to be a crucial regulator of Wnt signal throughput.

The loss of FrzB expression in gastric cancer suggests that this sWA may act as a tumour suppressor, and that its loss in malignancy removes FrzB mediated inhibition of proliferative Wnt signals. Our data are consistent with an earlier report by To et $a l^{16}$ which described downregulation of this sFRP in gastric cancers. A similar inactivation and downregulation of sFRPl, the expression of which was not investigated in our study, has been reported in colorectal carcinogenesis. ${ }^{17}$ Downregulation of some sWAs is also seen in cancers arising from the breast, cervix, and bladder. ${ }^{18-20}$ Thus, it appears that some sWAs act as tumour suppressors and the loss of sWAs may contribute to cancer development. Our data implicate FrzB in gastric carcinogenesis.

The gastric cancer specimens analysed ranged from well differentiated to poorly differentiated and included intestinal, diffuse, and signet ring histologies. No correlation between histological subtype or grade and sWA expression was seen. The colon cancer specimens were predominately moderately or moderately to well differentiated. About half presented with lymph node involvement, but no correlation between sWA expression and locoregional lymph node involvement was seen. In a previous study in breast cancer, ${ }^{18}$ the number of axillary lymph nodes involved with tumour was significantly lower when primary tumours expressed high levels of 


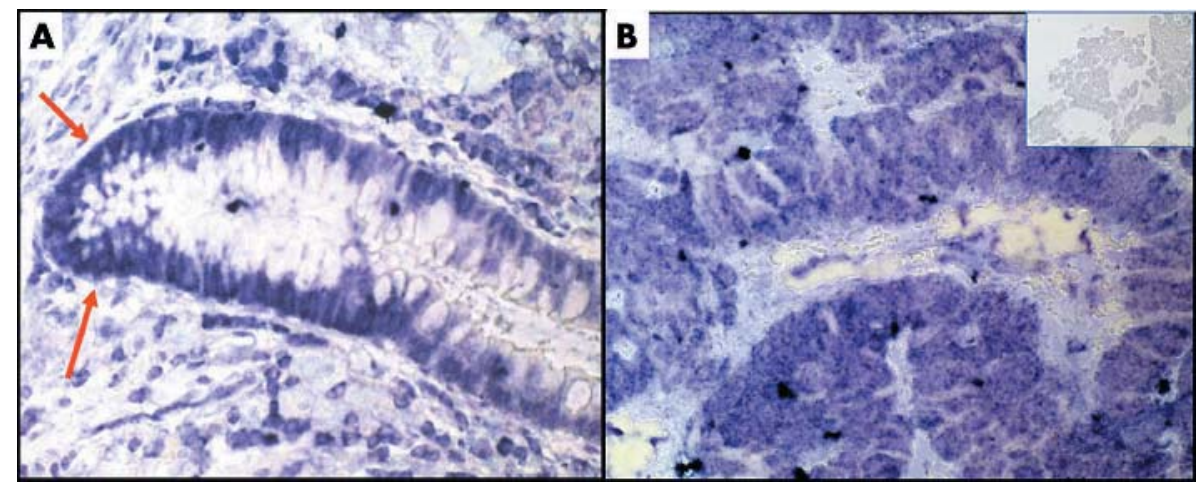

Figure 3 In situ hybridisation using an antisense Wif1 RNA probe in colon tissue samples. The purple colour indicates positive hybridisation. The insert shows the negative sense probe control. (A) Normal colon tissue. Note the positive hybridisation with relatively stronger staining intensity at the base of the crypts (arrows). Original magnification, $\times 40$. (B) Colon cancer tissue. Positive hybridisation. Original magnification, $\times 40$. Normal gastric and gastric cancer tissue showed no staining for Wifl (not shown).

frizzled related protein, and expression increased with increasing histological grade. In the gastrointestinal samples analysed here, no such correlations could be established.

We found that sWAs such as Dkk3 in gastric tissues and Wifl, Dkk2, Dkk3 in colon tissues are differentially expressed in the deep gastric glands/base of the colonic crypt compared with the superficial or mucosally oriented gastric glands/ mucosal aspect of the colonic crypt. Higher expression of sWA genes was seen at the base of colon crypts and in the deep gastric glands, areas where proliferative and undifferentiated gastrointestinal stem cells reside. ${ }^{21}$ Nuclear accumulation of $\beta$ catenin, a marker of canonical Wnt pathway activation, is specifically found in the epithelial cells located at the bottom of the colonic crypt. ${ }^{22}{ }^{23}$ The $\beta$ catenin-Tcf4 complex, and its target gene c-myc, has been proposed as a switch that regulates the balance between proliferation and differentiation in the intestinal epithelium. ${ }^{22}$ In other tissues, Wnt signalling has been shown to play a role in homeostasis and self renewal of normal haemopoietic stem cells, ${ }^{24}$ and purified Wnt3a has been shown to act directly as a stem cell growth factor. ${ }^{25}$ Wnt proteins also regulate stem cell function in the skin. ${ }^{26}$ Our data suggest that, in the gastrointestinal tract, one way in which Wnt signal throughput in the stem cell compartment is regulated is through differential expression of sWAs.

"It is possible that higher expression of Dkks, in particular Dkk3, in the area of the gastrointestinal stem cells promotes non-canonical signalling, and that these noncanonical signals are crucial in maintaining a proliferating, but undifferentiated stem cell pool"

The expression of sWAs may inhibit canonical Wnt signals, as suggested by Pinto et al, ${ }^{15}$ preventing differentiation of stem cells and thereby maintaining the stem cell pool. Dkks have been proposed to inhibit canonical Wnt signals primarily, possibly promoting signalling through non-canonical pathways. ${ }^{6}$ It is possible that higher expression of Dkks, in particular Dkk3, in the area of the gastrointestinal stem cells promotes non-canonical signalling, and that these noncanonical signals are crucial in maintaining a proliferating, but undifferentiated stem cell pool. Previous results from our laboratory ${ }^{8}$ described increased expression of Wnt5a at the base of the colonic crypts. Wnt5a is involved in non-canonical signalling through both the Wnt5a- $\mathrm{Ca}^{2+}$ pathway ${ }^{27}$ and the Wnt-Jnk pathway. ${ }^{28}$ Thus, the Wnt5a and Dkk data are consistent and suggest an important role for non-canonical
Wnt signals in the regulation and maintenance of gastrointestinal stem cells.

Although a differential, zonal distribution of Dkk3 has been described previously in the adrenal cortex, ${ }^{29}$ where Wnt signalling is also tightly regulated, the expression patterns of Wifl are less well characterised, although downregulation in some cancers has been reported. ${ }^{30}$ In addition, although Wif 1 binds to secreted Wnt ligands, ${ }^{31}$ its exact mechanism of action in inhibiting Wnt signals has not been defined. Wifl has no sequence similarity with the CRD domain of Fz or sWAs. In drosophila models, Wifl can bind to XWnt8 and drosophila Wg in the extacellular space and inhibit Xwnt8Dfz2 interactions and Armadillo stabilisation. Whether a similar inhibition of Wnt-Fz interactions occurs in mammalian cells is not clear. In addition, whether the zonal distribution pattern of Wifl seen in our study is linked to non-canonical Wnt signals, as proposed for Dkk3, requires further study.

An alternative model could be proposed if in human gastrointestinal tissues Dkk2 and Dkk3 do not exhibit Wnt antagonist activity but, instead, enhance Wnt signal throughput. Dkk3 does not suppress Wnt induced secondary axis formation in the xenopus embryo, ${ }^{32}$ and may act as a positive ligand for LRP5/6 to promote Wnt signals. Dkk2 is thought to act as a Wnt agonist via its interaction with LRP6. ${ }^{33}$ Thus, Dkk2 and Dkk3 may be acting as Wnt agonists, promoting

Take home messages

- The expression of soluble Wnt antagonists (sWAs) differs between the upper (gastric) and lower (colon) gastrointestinal tract

- The loss of the soluble frizzled related protein, FrzB, in gastric cancer suggests that it acts as a tumour suppressor

- The zonal expression of Dkk3 in gastric tissue, and Dkk2, Dkk3, and Wif1 in colon tissue, with increased expression in the deep gastric glands/colonic crypt bases, where gastrointestinal stem cells reside, suggests that sWAs may be crucial Wnt signalling regulators in these tissues, and may contribute to the maintenance of the stem cell pool

- sWAs appear to be important components of the gastrointestinal proliferative regulatory network 
Wnt dependent proliferation in the stem cell compartment. In our study, Dkk2 was expressed in a graded fashion in colonic tissues, but was not expressed in gastric tissues. The results suggest that Dkk2 may play some role in the maintenance of the stem cell compartment in the intestinal epithelium, but is not involved in gastric epithelial maintenance.

Homeostasis of the intestinal epithelium is strongly dependent on the balance existing between cell proliferation, cell cycle arrest, and cell migration. The Wnt signalling pathway is one important regulator of gastrointestinal stem cell proliferation and homeostasis. Our study indicates that the expression of sWAs is an important component of this regulatory network.

\section{ACKNOWLEDGEMENTS}

Supported by NIH grant CA-082452 and the Chao Family Comprehensive Cancer Center. The authors would like to thank Dr $\mathrm{M}$ Waterman for her input into the design of experiments and her critical review of this manuscript.

\section{Authors' affiliations}

T Byun, M Karimi, T Milovanovic, R F Holcombe, Division of Hematology/Oncology, University of California, Irvine Medical Center, 101 The City Drive, Bld 23, Rm 244, Orange, CA 92868, USA

J L Marsh, Department of Developmental and Cell Biology, University of California

F Lin, Department of Pathology, University of California

\section{REFERENCES}

1 Cadigan KM, Nusse R. Wht signaling: a common theme in animal development. Genes Dev 1997;11:3286-305.

2 Wells J, Melton D. Vertebrate endoderm development. Annu Rev Cell Dev Biol $1999 \cdot 15 \cdot 393-410$.

3 Polakis P. Wnt Signaling and cancer. Genes Dev 2000;14:1839-51.

4 Moon R, Bowerman B, Boutros $M$, et al. The promise and perils of Wnt signaling through beta-catenin. Science 2002;296:1644-6.

5 Huelsken J, Birchmeier W. New aspects of Wnt signaling pathways in higher vertebrates. Curr Opin Genet Dev 2001;11:547-53.

6 Zorn AM. Wnt signalling: antagonistic dickkopfs. Curr Biol 2001; 11:R592-5

7 Kawano Y, Kypta R. Secreted antagonists of the Wnt signaling pathway. J Cell Sci 2003; 116:2627-34

8 Holcombe RF, Marsh JL, Waterman ML, et al. Expression of Wnt ligands and Frizzled receptors in colonic mucosa and in colon carcinoma. J Mol Pathol 2002;55:220-6.

9 de Santa Barbara P, van den Brink GR, Roberts DJ. Development and differentiation of the intestinal epithelium. Cell Mol Life Sci 2003;60:1322-32.

10 Lickert H, Kispert A, Kutsch S, et al. Expression patterns of Wnt genes in mouse gut development. Mech Dev 2001;105:181-4.

11 Lee YJ, Swencki B, Shoichet S, et al. A possible role for the high mobility group box transcription factor Tcf- 4 in vertebrate gut epithelial cell differentiation. J Biol Chem 1999;274:1566-72.
12 Heller RS, Dichmann DS, Jensen J, et al. Expression patterns of Wnts, Frizzleds, sFRPs and misexpression in transgenic mice suggesting a role for Wnts in pancreas and foregut pattern formation. Dev Dyn 2002;225:260-70.

13 Theodosiou NA, Tabin CJ. Wnt signaling during development of the gastrointestinal tract. Dev Biol 2003;259:258-71.

14 Korinek V, Barker N, Moerer P, et al. Depletion of epithelial stem-cell compartments in the small intestine of mice lacking Tcf- 4 . Nat Genet 1998; 19:379-83

15 Pinto D, Gregorieff A, Begthel $H$, et al. Canonical Wnt signals are essential for homeostasis of the intestinal epithelium. Genes Dev 2003;17:1709-13.

16 To KF, Chan MWY, Leung WK, et al. Alterations of frizzled (FzE3) and secreted frizzled related protein (hsFRP) expression in gastric cancer. Life Sci $2001 ; 70: 483-9$.

17 Caldwell GM, Jones C, Gensberg K, et al. The Wnt antagonist sFRPI in colorectal tumorigenesis. Cancer Res 2004;64:883-8.

18 Wong SC, Lo SF, Lee KC, et al. Expression of frizzled-related protein and Wnt-signalling molecules in invasive breast tumours. J Pathol 2002; 196: 145-53.

19 Ko J, Ryu KS, Lee YH, et al. Human secreted frizzled-related protein is downregulated and induces apoptosis in human cervical cancer. Exp Cell Res 2002;280:280-7.

20 Stoehr R, Wissmann C, Suzuki H, et al. Deletions of chromosome $8 p$ and loss of $\mathrm{sFRP1}$ expression are progression markers of papillary bladder cancer. Lab Invest 2004;84:465-78.

21 Brittan M, Wright NA. Gastrointestinal stem cells. J Pathol 2002; 197:492-509.

22 van de Wetering $M$, Sancho $E$, Verweij $C$, et al. The beta-catenin/TCF4 complex imposes a crypt progenitor phenotype on colorectal cancer cells. Cell 2002;111:241-50.

23 Batlle $E$, Henderson JT, Beghtel $H$, et al. $\beta$-Catenin and TCF mediate cell positioning in the intestinal epithelium by controlling the expression of EphB/ EphrinB. Cell 2002;111:251-63.

24 Reya T, Duncan AW, Ailles $L$, et al. A role for Wnt signaling in self-renewal of haematopoietic stem cells. Nature 2003;423:409-14.

25 Willert K, Brown JD, Danenberg E, et al. Wnt proteins are lipid-modified and can act as stem cell growth factors. Nature 2003:423:448-52.

26 Alonso L, Fuchs E. Stem cells in the skin: waste not, Wnt not. Genes Dev 2003; 17:1 189-200.

27 Ishitani T, Kishida S, Hyodo-Miura J, et al. The TAK1-NLK mitogen-activated protein kinase cascade functions in the $\mathrm{Wnt} 5 \mathrm{a} / \mathrm{Ca}+2$ pathway to antagonize Wnt/ $\beta$-catenin signaling. Mol Cell Biol 2003;23:131-9.

28 Yamanaka $\mathrm{H}$, Moriguchi T, Masuyama N, et al. JNK functions in the noncanonical Wnt pathway to regulate convergent extension movements in vertebrates. EMBO Rep 2002:3:69-75.

29 Suwa T, Chen M, Hawks CL, et al. Zonal expression of dickkopf-3 and components of the Wnt signaling pathways in the human adrenal cortex. J Endocrinol 2003;178:149-58.

30 Wissmann C, Wild PJ, Kaiser S, et al. WIF1, a component of the Wnt pathway, is down-regulated in prostate, breast, lung and bladder cancer. J Pathol 2003;201:204-12.

31 Hshieh JC, Kodjabachian L, Rebert ML, et al. A new secreted protein that binds to Wnt proteins and inhibits their activities. Nature 1999;398:431-6.

32 Krupnik VE, Sharp JD, Jiang C, et al. Functional and structural diversity of the human Dickkopf gene family. Gene 1999;238:303-13.

33 Li L, Mao J, Sun L, et al. Second cysteine-rich domain of Dickkopf-2 activates canonical Wnt signaling pathway via LRP-6 independently of disheveled. J Biol Chem 2002;277:5977-81.

34 Brott BK, Sokol SY. Regulation of Wnt/LRP signaling by distinct domains of Dickkopf proteins. Mol Cell Biol 2002;22:6100-10. 